\title{
Proposta de um modelo de processo para o desenvolvimento de cenários
}

Claudia Pedroso Silva, Jorge Luis Nicolas Audy

Pontifícia Universidade Católica do Rio Grande do Sul - PUCRS

Faculdade de Informática - Programa de Pós-Graduação em Ciência da Computação

Av. Ipiranga, 6681 - Prédio 16 - Porto Alegre, RS - CEP 90619-900

\{cpsilva; audy\}@inf.pucrs.br

Abstract. The comprehension of the organization on different performance environments is important because this knowledge permit an evaluation and a better vision about it actions. The organizations search models and techniques to understand these environments. The construction of scenario is used to make a map the external and internal environment of the organizations. The use of the scenario technique by organization permits to formulate of strategies and implement the actions minimizing uncertainties and risks. The aim of this paper is propose a process of scenario development and identifying the potential contributions this technique. This study is characterized as exploratory, since the main research method was the case study. A proposal of model of process for the scenario development is presented as results.

Key Words: Scenario, model, process

Resumo. O entendimento da organização sobre os diferentes ambientes onde atua ê importante, pois este conhecimento possibilita uma avaliação e um melhor direcionamento das suas ações. Para tanto, as organizações buscam modelos e técnicas para entender estes ambientes. A construcão de ceńrios tem sido usada para mapear para enter an a iente e a implementação de ações para a minimização de incertezas e riscos. O objetivo deste artigo é propor um processo para o desenvolvimento de cenários, baseado na análise feita a partir do uso desta técnica no contexto de uma organização de desenvolvimento de software. Este estudo pode ser considerado como exploratório sendo que o principal método de pesquisa foi o estudo de caso. Como resultado, apresenta-se além da proposta também as contribuições potenciais da técnica.

Palavras Chaves: Cenários, Modelo, Processo

1. Introdução

As transformações econômicas, políticas, sociais e tecnológicas que vem ocorrendo na sociedade causaram profundas mudanças nos cenários nacional e internacional. Tai transformações levaram as organizações a mudarem. O conhecimento do ambiente externo e interno torna-se uma necessidade à medida que começa a impactar diretamente nos negócios. Assim, as organizações estão buscando alternativas para anteciparem acontecimentos e estarem preparadas para as mudancas.

$\mathrm{O}$ entendimento da organização sobre os diferentes ambientes onde atua tornase importante, pois este conhecimento possibilita uma avaliação e um melhor direcionamento das suas ações. A construção de cenários tem sido usada para mapear o ambiente externo e interno das organizações, possibilitando a formulação de estratégias e a implementação de ações para a minimização de incertezas e riscos (Leemhuis, 1985).

Neste contexto, uma organização de desenvolvimento de software desenvolveu um estudo de cenários visando auxiliar a definição de suas estratégias. O objetivo deste artigo é propor um processo de desenvolvimento de cenários baseado na análise feita a partir do uso desta técnica no contexto da organização. Este estudo pode ser considerado 
como exploratório, sendo que o principal método de pesquisa foi o estudo de caso. Como resultado, o artigo apresenta uma proposta de modelo de processo para. desenvolvimento de cenários e identifica as contribuições potencio de processo para $\mathrm{O}$ artigo está estruturado da seguinte forma: cenários e sua aplicabilidade. A seção 3 apresenta 0 étoção 2 trata-se da técnica de trabalho e tam utilizado neste trabalho e também discorre sobre o estudo de caso do processo de construca neste cenários. Na seção 4, apresenta-se o modelo proposto para onstrução de desenvolvimento de cenários. A seção 5 traz as considon para o fim, são apresentadas as referências bibliográficas utilizadas neste texto. proposta. Por

\section{A Técnica de Cenários}

Historicamente, a técnica de cenários foi introduzida pela disciplina de planejamento militar e, subseqüentemente, adotada em várias outras áreas, tais como economia gerência e planejamento (Becker,1983). Nas últimas décadas sua utilizacãa tem alastrado por várias outras áreas, entre elas; Planejamento Estratégico, onde se utilizados na exploração de alternativas futuras, Planejamento Estratégico, onde são utilizados tanto na descrição de problemas como emo Carroll, 1995; Ringlocicon som Quanc Ringland,

Quando se trabalha o conceito de cenários, remete-se para conceitos que envolvem previsão do futuro, prospecção e planejamento. A técnica consiste que construção de futuros alternativos, através da análise de mútuas influências de fatores econômicos, sociais, políticos, culturais e tecnológicos de mutuas influências de fatores economicos, sociais, politicos, culturais e tecnológicos que retratam o ambiente externo e interno relevante para a atuação de uma organização (Heijden, 1996). Boar (1993) em clareza a questão da incerteza conceituando a construção de cenários como uma maneira estruturada de definir futuros possíveis, entendendo as possibilidades de cada possível futuro e desenvolvendo alternativas para as incertezas.

A construção de cenários contribui na prospecção dos ambientes interno externo a organização, auxiliando a tomada de decisão e a definição das estratégias ações desta. A proposta desta técnica está centrada em acontece se?" (Sprague, 1999) Wack (1985b) salinta questar contemplar eventos que possam ser controláveis; possibilitar a captura de diferentes pontos de vista; estimular o debate, possibilitando o aprendizado, o questionamento e mudanças de modelos mentais. Os principais elementos de um cenário que devem ser contemplados para auxiliar o entendimento e a construção dos mesmos são: o ambiente empresarial, o governo, a sociedade, as pessoas e a tecnologia.

Os cenários devem ser tanto exeqüíveis quanto surpreendentes, devem ter a capacidade de romper com estereótipos e serem elaborados da forma participativa traduzindo-se em mundos possíveis e caminhos alternativos. Porém, tão importante quando os cenários é o seu processo de construção. O processo deve ser observado, pois é este que irá viabilizar a construção e validar a veracidade, aplicabilidade e exeqüibilidade dos cenários (Millett, 1988).

\section{Método de Pesquisa}

Esta pesquisa caracteriza-se como exploratória, pois se procurou desenvolver proposições, aprofundar e buscar conceitos. Tem como seu principal método de pesquisa o estudo de caso que, segundo Yin (2001), pode ser definido como uma investigação empírica de um fenômeno dentro de seu contexto real.

O estudo foi desenvolvido em uma organização de grande porte da área de software. A empresa possui diversas filiais ao redor do mundo, tendo uma forte atuação no mercado. O principal negócio da organização estudada é o desenvolvimento e aperfeiçoamento de software para suportar as demais áreas de negócios.

\subsection{O Processo de Construção de Cenários da organização estudada}

processo de construção de cenários teve como objetivo possibilitar à organização o melhor entendimento sobre os seus ambientes e sobre o seu negócio, listando os seus principais fatores críticos de sucesso e organizando-os em cenários possíveis. O estudo contou com a participação de colaboradores da Organização.

Este processo constituiu-se de três etapas: Etapa Preparatória, Etapa de Desenvolvimento e Etapa de Consolidação. A Etapa Preparatória envolveu um conjunto de atividades preliminares que foram gerenciadas pela área de treinamento da organização e pela equipe de pesquisa. Nesta etapa definiu-se a agenda de trabalhos, o grupo de participantes, a metodologia de trabalho, os contatos com um pesquisador visitante e o levantamento e a pesquisa bibliográfica sobre a técnica de cenários.

Como resultados selecionou-se o grupo que participaria da Etapa de Desenvolvimento. Este grupo foi constituído pelo Diretor, pelos Gerentes e pelo Grupo de Processos e Qualidade. Como instrutor e mediador da Etapa de Desenvolvimento e Consolidação, atuou um pesquisador visitante especialista na técnica de cenários. Como insumos para a Etapa de Desenvolvimento, a primeira etapa gerou: a agenda e o escopo do trabalho, o material de apoio e o convite para os participantes.

A Etapa de Desenvolvimento constituiu-se de duas fases: o Nivelamento Conceitual e Metodológico e a Geração de Cenários. A fase de Nivelamento Conceitua e Metodológico iniciou com o Pesquisador visitante apresentando a técnica de cenários. Logo após essa conceituação, estabeleceram-se as primeiras discussões sobre tendências e perspectivas de interesse da organização e que cenários poderiam ser obtidos com este trabalho. Esta fase encerrou-se com a explicação dos procedimentos e técnicas que seriam utilizadas na próxima fase da Etapa de Desenvolvimento.

A fase 2 da Etapa de Desenvolvimento, que foi chamada de Geração dos Cenários, constitui-se de duas sub-fases: o Mapeamento da Organização e seu Ambiente e o Desenvolvimento dos Cenários. A primeira sub-fase estava relacionada ao Mapeamento da Organização e do seu Ambiente, no intuito de conhecer os fatores que poderiam afeta-lá. Como resultado deste mapeamento buscou-se a identificação das forças direcionadoras que eram consideradas fundamentais para o negócio. A segunda sub-fase, o Desenvolvimento dos Cenários, tratou do método utilizado para a construção dos cenários, da análise dos resultados e do monitoramento desse processo.

\section{Sub-Fase 1: Mapeamento do Ambiente e da Organização}

O mapeamento foi constituído de três atividades que propiciaram uma análise, realizada pelo grupo de participantes do processo, dos principais fatores que seriam relevantes no contexto da organização e que seriam os insumos para a próxima atividade.

As três atividades do mapeamento são: a Identificação das Oportunidades, Ameaças, Forças e Fraquezas; a Seleção dos Principais Fatores que impactam na organização e a Construção do Mapa Conceitual.

Atividade 1: Identificação das Oportunidades, Ameaças, Forças e Fraquezas.

Nesta primeira atividade, a tarefa constituiu-se de um debate sobre oportunidades e ameaças ambientais; potencialidades e vulnerabilidades da organização, através da técnica de brainstorming, Esta técnica é uma dinâmica de grupo que visa a produção de idéias novas sobre um assunto, livres de qualquer restrição. 
Primeiramente, elencou-se as oportunidades, logo após as ameaças, forças e fraquezas. Cada participante dava uma sugestão de oportunidade até que todos se manifestassem ou não houvesse mais sugestões. O mesmo procedimento foi adotado para cada um dos demais itens. A partir da identificação de cada um destes itens foi realizada uma seleção para cada item daqueles que seriam mais importantes. Ou seja, quais as oportunidades mais importantes, quais as ameaças mais importantes, quais as forças mais importantes e quais as fraquezas mais importantes. Foi realizada uma eleição para estas escolhas. Essa atividade deu origem a matriz SWOT (Strengths, Weaknesses, Opportunities, Threats). Um exemplo dessa matriz é apresentado no Quadro 1.

Quadro 1. Matriz SWOT

\begin{tabular}{|c|c|c|c|}
\hline Oportunidades & Ameaças & Forças & Fraquezas \\
\hline Processos & Cenário Mundial & Agillidade & Imagem \\
Treinamento & Novas Tecnologias & Qualidade & Diferença Cultural \\
Comunicação & Custo Brasil & flexibilidade & Concorrentes \\
\hline
\end{tabular}

\section{Atividade 2: Seleção dos Fatores Críticos de Sucesso}

Partindo do conjunto de fatores gerados pela Matriz SWOT, o grupo procurou refletir sobre cada um desses fatores e agrupá-los em um conjunto que reunia as principias oportunidades, ameaças, forças e fraquezas. $\mathrm{O}$ processo foi interativo, permitia idas e vindas até que os arranjos finais fossem os melhores possíveis. O grupo analisou cada conjunto de itens e verificou quais foram os fatores que mais impactavam a organização gerando um único grupo de fatores. O conjunto desses Fatores foi o insumo para a Construção do Mapa Conceitual.

\section{Atividade 3: Construção do Mapa Conceitual}

Com base na seleção de fatores, construiu-se o mapa conceitual. Mapas Conceituais são representações gráficas semelhantes a diagramas, que indicam relações entre conceitos ligados por palavras. Representam uma estrutura que vai desde os conceitos mais abrangentes até os menos inclusivos (Ausubel, 1980).

Esse mapa tinha como objetivo demonstrar como os fatores estavam interrelacionados e qual era o impacto desses fatores um sobre os outros. Os relacionamentos e a atribuição das cardinalidade foram dadas a partir das discussões do grupo. Cabe salientar que os fatores estavam presentes no mapa foram decorrentes das decisões anteriores geradas nas outras duas atividades.

\section{Sub-fase 2: Desenvolvimento dos Cenários}

A sub-fase 2 foi composta por três atividades: a Construção dos Cenários, a Análise dos Cenários Construídos e a Monitoramento desses Cenários.

\section{Atividade 1: Construção dos Cenários}

$\mathrm{Na}$ construção dos cenários, a proposta foi estruturar cenários utilizando o método indutivo. Para cada cenário que foi desenvolvido, utilizou-se os fatores encontrados no mapa conceitual como entrada para a descrição de uma história que envolvesse esses fatores. Nesta história foram criados atores e estimou-se um tempo para o evento. Assim, a primeira tarefa, foi criar cartões de eventos com a descrição dos atores envolvidos e uma data possível. Essa data não poderia ser superior a um ano (tempo estipulado como ideal para a tarefa proposta).

Cada cenário que foi criado estava associado a um conjunto de eventos. O grupo criou eventos que estavam ocorrendo e que estavam relacionados aos fatores do
Mapa Conceitual. Os eventos referiam-se ao macroambiente e ao microambiente que fletissem o negócio de maneira a formar a base para o processo de geração de trácias. Os eventos tinham reflexos em diversos elementos do mo concorrentes, fornecedores, infra-estrutura, tecnologia, entre outros. Toda a criação de crocesso interativo, onde as opiniões eram discutidas, antes eventos realizou-se num prepetidos em mais de um que fossem incluídos no cenário. Alguns eventos reqüência cronológica.

cenário, dada a restrição que esses deveriam seguir uma sequencia crórios. Aiscussão do Com os eventos criados passou-se à construção dos cenários. A discussão do grupo concentrou-se em como realizar a distribuição destes eventos de forma a torna exeqüíveis. Esses cenários deveriam demonstrar um equilíbrio entre os eve exequivers. não deveriam ser criados cena história foi montada para cada cenário e a partir dela

puderam ser encaminhadas algumas ações.

\section{Atividade 2: Análise dos Cenários Construidos}

grupo analisou os cenários e as histórias A contadas, promovendo uma conticar a validade do concentrados esforços de melhoramento. Nesta atividade para questões: "o por quê isso cenário construído, o cenário deveria responder a duas ques cenario cons " "o que aconteceria a partir dai". Respondendo a essas duas perguntas aconteceria" co que acontecerado como adequado.

\section{Atividade 3: Monitoramento dos Cenários}

dos, os cenários teriam a função de atuar como parte do processo de para que a técnica seja efetiva são necessários ajustes ao longo do , para que a técnica seja enários tempo. A atividade de Monitoramento representou esta prosim o planejamento e deviam ser revis

processo de construção

Na Etapa de Consolidação se deu o fechamento ao processo de construção dos cenários. Nesta etapa foi realizada a avaliação sobre todo processo desenvolvido. A avaliação levantou questões sobre a aplicabilidade, contexto de uso e técnicas para a implementação de cenários no dia-a-dia da organização e formas de divulgação deste trabalho.

O processo de construção de cenários da organização teve um tempo total de proximadamente três semanas. A etapa que mais consumiu tempo foi a Preparatória. 位e As Etapas de Desenvolvimento e Consolidação foram etapas de imersão ondado a criação as atividades foram realizadas num total de três dias e tiveram como

de cenários plausíveis para o ambiente de atuação da organização.

4. Proposta de um Modelo de Processo de Desenvolvimento de Cenários (descrito na Tendo por base a aprendizagem resultante do estudo de caso desenvolvido (descrito na Teñ 3 .1) buselo de processo para o desenvolvimento de seção 3.1), buscou-se propor úc descrição do processo já envolveu a sistematização cenários. Destaca-se que a propria descrição do processo ja envesenta-se o modelo de por parte do grupo de pesquisa do modelo proposto. A seguir, apresenta-se a modes. por parte do grescricão de suas etapas, 1) modelo é o resultado da análise do processo como um todo, desde a cenários.

sua conclusão, e do detalhamento da sub-fase de desento

O modelo é composto por três etapas, que são: Etapa Preparatoridida em Desenvolvimento e

atividades e/ou fases. 


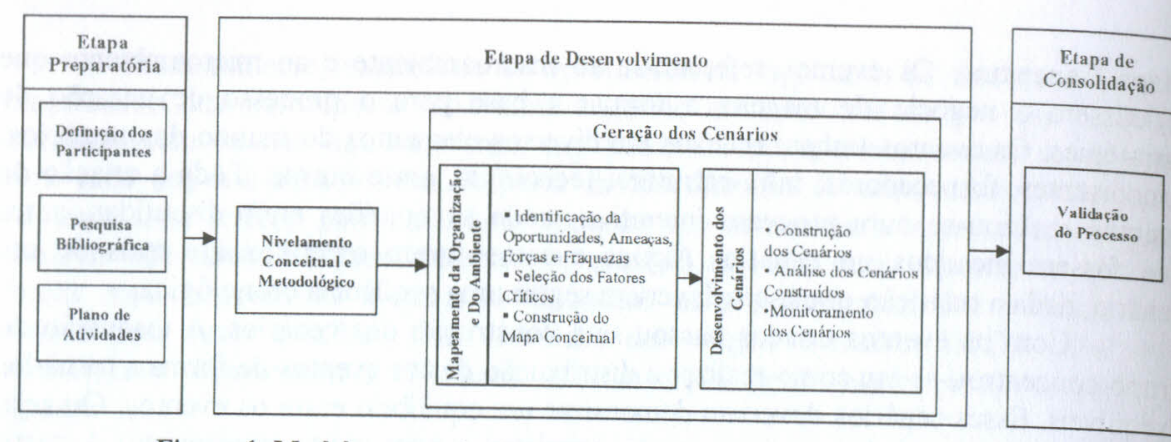

Figura 1. Modelo proposto para processo de desenvolvimento de cenários

A Etapa Preparatória tem por objetivo organizar e preparar as demais etapas. Cabe à esta etapa a definição dos participantes do processo de construcão de cenários. busca de referências teóricas e práticas para o desenvolvimento das atividades. Só então se parte para a elaboração do Plano de Atividades a serem desenvolvidas nas etapas subseqüentes do processo (Etapas de Desenvolvimento e Consolidação). A definição dos participantes é uma das atividades mais importantes, pois essa seleção irá impactar diretamente na riqueza dos cenários construídos. Quanto maior for à diversidade dos membros envolvidos no processo, maior será o número de opiniões presentes na sua construção. A busca de referências teóricas e práticas é uma atividade que tem por objetivo promover um elo de ligação entre a teoria e a prática, visando que o processo realizado seja metodologicamente válido. No Plano de Atividades que estarão presentes todos os detalhes do processo a ser realizado. Esse detalhamento envolve cronogram escopo e custos. O plano deve contemplar também uma previsão de tempo de preparação e treinamento a ser utilizado para cada etapa e atividade, visto que como se trabalha com grupos heterogêneos, muitas vezes, é necessário um nivelamento de conhecimentos antes da continuidade do processo de desenvolvimento de cenários.

O resultado da Etapa Preparatória é a organização do processo de desenvolvimento de cenários. Essa preparação é importante à medida que vai favorecer o planejamento das demais etapas, organizando o processo de desenvolvimento.

A Etapa de Desenvolvimento é o ponto central do modelo. É onde serão desenvolvidos os cenários. A etapa possui duas fases, sendo elas o Nivelamento Metodológico e Conceitual e a Geração de Cenários.

No Nivelamento, será verificado se existe uma uniformidade de conhecimento sobre a técnica e sua aplicação. Caso exista algum desnivel, é nesta fase que essas questões devem ser levantadas e identificadas. A partir desta identificação, será avaliada a necessidade de treinamentos sobre os conceitos e a metodologia (estas horas devem estar estimadas no plano de atividades da etapa anterior). O Nivelamento, além proporcionar o treinamento, iniciará as discussões sobre tendências e problemas para a organização que terá sua intensificação na próxima etapa, que é a Geração dos Cenários. A importância desta fase está em propiciar a todos os participantes um conhecimento uniforme da técnica, possibilitando que sua aplicação seja melhor conduzida.

A Geração dos Cenários é composta por duas sub-fases: o Mapeamento da Organização e do Ambiente e o Desenvolvimento dos Cenários. Cada uma dessas subfases tem um conjunto de atividades específicas conforme foi observado no estudo de caso.
A sub-fase de Mapeamento tem por objetivo levantar as tendências globais e nacionais de interesse da organização envolvida neste processo. Este mapeamento deverá fornecer uma visão sobre os principais fatores impactadores tanto internamente quanto externamente da organização. Nesta sub-fase serão coletados os dados e as informações relativas aos acontecimentos que movem a organização e aos acontecimentos ambientais de relevância para esta. Inicia-se também a identificação das conexões entre os fatores, procurando demonstrar quais são, dentre os importantes, os mais importantes. A idéia desta sub-fase é clusterizar até obter-se um pequeno número que são os fatores determinantes. A partir desta identificação, as conexões são estabelecidas com o auxílio de técnicas de representação gráficas (mapas conceituais), favorecendo a visualização das inter-relações dos fatores.

A sub-fase de Desenvolvimento de Cenários envolve as atividades de construção, análise e monitoramento dos cenários. Nesta fase se faz uso de métodos para o desenvolvimento dos cenários. Para este modelo, optou-se por utilizar o método indutivo devido a maior flexibilidade deste, que permite uma maior interação entre as pessoas participantes do grupo de construção. O método indutivo parte de uma lista de eventos para a construção de uma estrutura maior, que são os cenários. Com o auxílio dos fatores identificados e do mapa conceitual gerado são criados os eventos e acrescentados novos fatores que podem ser mapeados ao longo do processo, visto que este é um processo interativo. Após a criação dos eventos, o próximo passo do método é a construção dos cenários. Os eventos são organizados em cenários possíveis, montando-se uma breve história com os eventos selecionados estabelecendo-se conexões lógicas e consistentes entre estes. Os participantes podem criar novos eventos, caso necessário, conforme o cenário for sendo construído.

Após a Construção dos Cenários passa-se para a Análise e o Monitoramento. Estas atividades são críticas no que tange a participação dos grupos e a interação entre as três atividades. Da análise e do monitoramento poderão ser concebidas as estratégias da organização. A análise dos cenários construídos envolve a validação destes cenários. Essa atividade testa a consistência e proporciona uma visualização dos pontos que podem estar fracas e que podem representar ameaças para a organização.

Com os cenários construídos e validados, é necessário se estabelecer um Monitoramento. Essa atividade é importante, pois evita que os cenários tornem-se obsoletos dentro de um contexto de mudanças constantes em que estão inseridas as organizações. Para esse monitoramento, da equipe que participou da construção dos cenários, seleciona-se um subgrupo que ficará responsável por acompanhar e reformular os cenários pelo tempo estipulado por estes.

Por fim, a Etapa de Consolidaçao é composta por uma atividade, a validação do processo, que tem por objetivo verificar como transcorreu o processo. Nesta etapa, avalia-se a aplicabilidade no contexto da organização e se os objetivos das etapas, das fases e das atividades chegaram ao resultado esperado. A Etapa de Consolidação faz o papel de controle de qualidade do processo.

\section{Considerações Finais}

Ao aplicar-se o modelo de processo proposto, tendo em vista a experiência do estudo de caso desenvolvido, identificou-se o conjunto de contribuições que demonstram a efetividade do uso da técnica em um contexto real. O processo possibilitou um melhor conhecimento do negócio através da identificação do conjunto de fatores relevantes para a atuação da organização, ou seja, aqueles que foram considerados como críticos foram 
explicitados e dispostos em cenários. No processo puderam ser visualizadas as possíveis variações nos fatores que poderiam afetar os negócios da organização. Através da análise realizada com a identificação dos fatores, das suas inter-relações e do seu impacto permitiram aprofundar o conhecimento sobre o ambiente analisado, propiciando assim um melhor entendimento desse ambiente externo.

O envolvimento do grupo na construção dos cenários proporcionou capacitação e treinamento teórico e prático ao pessoal, que ficou encarregado pela implementação e revisão dos cenários criados. Com relação aos resultados do estudo de caso, deve-se destacar as limitações típicas deste tipo de método de pesquisa no tocante a generalização dos resultados. A parte empírica deste estudo envolveu uma organização de desenvolvimento de software. Entretanto, pode-se utilizar o princípio de generalização analítica proposta por Yin (2001) que propicia um certo grau de generalização dos resultados ao constatar que a teoria é claramente confirmada pelos resultados do caso estudado.

O papel fundamental dos cenários para os dirigentes responsáveis pela formulação das estratégias de uma organização é servir como ferramenta para aprimorar o processo de planejamento e gestão ao explorar um conjunto de situações do tipo "e se isso acontecer". Pensar cenários é ensaiar antes do futuro, é estar preparado para aprender com o presente. O modelo visa auxiliar no processo de construção de cenários buscando formalizar a elaboração destes.

\section{Referências Bibliográficas}

AUSUBEL, D. et al. Psicologia Educacional. Editora Interamericana, New York, 1980

BECKER, H.A. The role of gaming and simulation in scenario project. Operational Gaming: an international approach. International Institute for Applied Systems Analysis, Luxemburg, Austria, 1983

BOAR, B. The Art of Strategic Planning for Information Technology. John Wiley \& Sons, Inc. USA, 1993.

CARroll, J. M. Scenario Based Design: Envisioning Work and Technology in System Development - John Wiley and Sons. USA, 1995

HEIJDEN, KEES VAN DER. Scenarios: the art of strategic conversation. John Wiley \& Sons, Inc. USA, 1996.

LEEMHUIS, J. P. Using Scenarios to Develop Strategies. Long Range Planning, V 18, N 2, 1985.

MILLET, S. "How Scenarios Trigger Strategic Thinking". Long Range Planning, V. 21 N. 5 , 1988.

RINGLAND, G. Scenario Planning: Managing for the future. John Wiley and Sons. USA, 1998.

SPRAGUE, R.H; McNURLIN, B.C. Information Systems Management in Practice. Canadá, Prentice Hall, 1999

WACK, P. Scenarios: Uncharted Waters Ahead. Harvard Business Review, September/October, 1985a

WACK, P. Scenarios: Shooting the Rapids. Harvard Business Review, November/December, $1985 b$

YIN, R. K. Estudo de Caso: Planejamento e Métodos. Porto Alegre: Bookman, 2001. 